\title{
Abundancia, tamaño y estructura poblacional del tiburón punta blanca de arrecife, Triaenodon obesus (Carcharhiniformes: Carcharhinidae), en Bahía Chatham, Parque Nacional Isla del Coco, Costa Rica
}

\author{
Ilena Zanella ${ }^{1}$, Andrés López-Garro ${ }^{1}$, Geiner Golfín-Duarte ${ }^{2} \&$ Joel C. Saenz ${ }^{3}$ \\ 1. Asociación Conservacionista Misión Tiburón, Playas del Coco, Guanacaste, Costa Rica; izanella@misiontiburon.org; \\ alopez@misiontiburon.org \\ 2. Parque Nacional Isla del Coco. Sistema Nacional de Áreas de Conservación, Ministerio del Ambiente, Energía y \\ Telecomunicaciones, San José, Costa Rica; geiner.golfin@sinac.go.cr \\ 3. Instituto Internacional en Conservación y Manejo de Vida Silvestre, Universidad Nacional de Costa Rica, Heredia, \\ Costa Rica; jsaenz@una.ac.cr
}

Recibido 28-III-2012. C Corregido 05-VII-2012. Aceptado 24-IX-2012.

\begin{abstract}
Abundance, size and population structure of whitetip reef sharks, Triaenodon obesus (Carcharhiniformes: Carcharhinidae), in Bahía Chatham, Parque Nacional Isla del Coco, Costa Rica. Approximately 12 species of shark inhabit at Coco Island National Park (PNIC); some are migratory, such as the hammerhead shark (Sphyrna lewini) and whale shark (Rincodon typus), and others are resident, like the whitetip shark (Triaenodon obesus). The whitetip shark is a species related to coral reefs, it inhabits coastal environments and is nocturnal and, it is very common in tropical waters, but there are few studies worldwide. In fact, only short researches have been done about their behavior at PNIC. Therefore, this study aimed to give data about the abundance, size and population structure of the whitetip shark at Chatham Bay in Coco Island National Park, providing baseline information for monitoring its population which is also very vulnerable to climate change. In order to accomplish this aim, a mark-recapture method was used, as well as underwater visual counts to determine their relative abundance and visual recaptures at Chatham Bay. The most effective hours for tagging were between 18:00 and 22:00 (sunset), when the whitetip sharks were more active probably related to foraging behavior. In addition, the depth was a relevant factor for tagging, sharks were caught in shallow water $(10.9 \pm 1.9 \mathrm{~m})$. This species prefers to feed in shallow water and this behavior is probably related to the distribution and occurrence of rock and coral reefs in Chatham Bay. Based on the results, whitetip shark do not have a defined spatial ontogenic segregation, since in the same sites both juveniles and adults were found. The study confirmed that whitetip shark use specific residence sites: during the tagging trips, different individuals were recaptured; and during the underwater counts, at least $32.76 \%$ of tagged sharks were observed. The average length of the tagged sharks was $101.8 \pm 12.1 \mathrm{~cm}(130.0 \mathrm{~cm}$ maximum and $71.0 \mathrm{~cm}$ minimum). The relative abundance of the whitetip shark in Chatham Bay observed was estimated on $49.5 \pm 10.4$ sharks/hour. Furthermore, using the recapture data obtained during the night tagging expeditions, a population of 408 (IC $=181-1050)$ sharks was estimated. Citation: Zanella, I., A. López-Garro, G. Golfín-Duarte \& J.C. Sáenz. 2012. Biología del tiburón punta blanca de arrecife, Triaenodon obesus (Carcharhiniformes: Carcharhinidae), en Bahía Chatham, Parque Nacional Isla del Coco, Costa Rica. Rev. Biol. Trop. 60 (Suppl. 3): 339-346. Epub 2012 Dec 01.
\end{abstract}

Key words: Parque Nacional Isla del Coco, Triaenodon obesus, Bahía Chatham, shark tagging, underwater visual counts, Cocos Island, sharks, Costa Rica.

El Parque Nacional Isla del Coco (PNIC) es reconocido a nivel mundial por la alta diversidad íctica, encontrándose entre sus arrecifes y aguas costeras más de 270 especies de peces. Por su condición de isla oceánica posee una alta cantidad de especies endémicas (27) y otras (20) que solo comparte con las Islas Galápagos (Ecuador) y la Isla Malpelo (Colombia) (Garrison 2005). Además, en el PNIC se han registrado 12 especies de tiburones que 
habitan o visitan periódicamente sus aguas, sobresalen especies migratorias como el tiburón martillo (Sphyrna lewini) y el tiburón ballena (Rincodon typus). Sin embargo, son comunes especies más locales como el tiburón punta blanca de arrecife (Triaenodon obesus) (Sibaja 2008). Durante años, esta riqueza marina ha atraído buzos y fotógrafos submarinos, por lo que actualmente el PNIC es uno de los sitios de buceo más importante y reconocido en el mundo (Onca Natural \& ACMIC 2007).

El tiburón punta blanca de arrecife aunque vive también en islas oceánicas, es una especie costera y de aguas tropicales, de hábitos bentónicos o demersales. Por lo general, descansa en cuevas durante el día, y actúa como un depredador activo nocturno, formando grupos para alimentarse en los arrecifes (Whitney et al. 2007). Se ha demostrado que en la noche permanece activo entre el $42 \%$ y el $67 \%$ de ese tiempo; mientras que en el día permanece poco activo, con periodos de actividad entre $10 \%$ y $24 \%$ (Whitney et al. 2007). Diferentes autores han informado sobre aspectos de la biología y ecología de la especie, tales como su distribución, presencia y abundancia (Randall 1977, Robbins 2006, Ayling \& Choat 2008, Lanyon 2011). Además, se ha comprobado que en PNIC, esta especie presenta una relación negativa con anomalías de la temperatura superficial del agua del mar (Sibaja 2008). Por lo tanto, parece ser muy sensible y vulnerable a anomalías de temperatura relacionadas a los cambios climáticos.

Es considerado un tiburón de talla mediana, con una longitud máxima reportada de $213 \mathrm{~cm}$ (Compagno et al. 1995). Al igual que la mayoría de tiburones actuales, es una especie vivípara cuyas hembras poseen un periodo de gestación aproximado de 11 meses. Debido a su estrategia vivípara las hembras de tiburón punta blanca de arrecife paren cada dos años un promedio de dos crías, con una talla de nacimiento de aproximadamente $60 \mathrm{~cm}$ (Robbins 2006).

En la actualidad es una de las especies más abundantes en el PNIC, donde probablemente habita la población menos perturbada del mundo. Esto la ha convertido en una atracción para el turismo, en especial cuando forrajean en el arrecife. A pesar de ser una especie muy común en aguas tropicales y de su importancia turística, pocos son los estudios biológicos y ecológicos que existen, como abundancia, reproducción, crecimiento y longevidad (Robbins 2006). En el PNIC, existe poca información sobre este tiburón $\mathrm{y}$, los estudios que existen fueron enfocados en aspectos de su comportamiento (Whitney et al. 2004, Whitney \& Motta 2007). Por lo anterior, los objetivos de este estudio fueron aportar datos sobre la abundancia, tallas y estructura de la población del tiburón punta blanca de arrecife en la zona de Bahía Chatham, PNIC.

\section{MATERIALES Y MÉTODOS}

Área de estudio: La Isla del Coco se ubica en aguas oceánicas del Pacífico costarricense $\left(5^{\circ} 32^{\prime} \mathrm{N}, 87^{\circ} 04^{\prime} \mathrm{W}\right)$ y fue declarada Parque Nacional en 1978. Su creación responde a proteger y conservar la biodiversidad terrestre y marina de esta singular isla oceánica (Cortés 2008). A su vez el PNIC fue inscrito en 1997 como Sitio Patrimonio Mundial, justificando su nomina en los hábitats críticos que presenta el área para la vida marina, en especial para las especies pelágicas, particularmente los tiburones (ACMIC 2002). El área de estudio se ubicó en Bahía Chatham (entre las coordenadas $5^{\circ} 33^{\prime} 25.28^{\prime \prime} \mathrm{N}, 87^{\circ} 02^{\prime} 53.11^{\prime \prime} \mathrm{W}$ y 5 33'04.71'”N, $\left.87^{\circ} 02^{\prime} 13.76^{\prime} \mathrm{W}\right)$, que es semi-encerrada entre dos puntas: Punta Agujas y Punta Pacheco. Al tomar como referencia dichas puntas, la Bahía Chatham tiene un ancho de $1350 \mathrm{~m}$ y $610 \mathrm{~m}$ de fondo. Distintas quebradas desembocan en la Bahía, sin embargo la principal entrada de agua dulce proviene del Río Chatham, ubicado en el sector central de la misma. El fondo de la Bahía se caracteriza por la presencia de distintas acumulaciones de rocas y arrecifes, aunque en la Bahía predomina el fondo arenoso con un fuerte cambio batimétrico o de profundidad (Sinergia 69 2002). 
Marcaje de tiburones: Se realizaron dos expediciones al PNIC para marcar tiburones. La primera del 16-30 diciembre 2010 y la segunda del 20-30 marzo 2011. Durante ambas expediciones se capturaron y marcaron tiburones punta blanca de arrecife con marcas convencionales tipo "flexi-tags" en Bahía Chatham. Se realizaron recorridos entre las 17:00-24:00h a bordo de una embarcación dotada de equipo para realizar las capturas de los tiburones. Los tiburones Se capturaron con una cuerda de mano en diferentes zonas y profundidades de la Bahía y, con anzuelos circulares \#5 (sin muesca para minimizar el daño físico en el tiburón), utilizando como carnada calamar gigante y calamar torpedo (Loliginidae). Una vez que los tiburones estuvieron enganchados, fueron subidos a la embarcación por medio de un "scoop" o "Dip Net", un tipo de cesta de red con una extensión metálica que permite su manipulación. Inmediatamente, se tomó la posición geográfica con un GPS, la hora de captura, profundidad de captura y temperatura superficial del agua.

A cada tiburón se le retiró el anzuelo, posteriormente se le evaluó el sexo (mediante la presencia o ausencia de los hemipenes), y fue medido con un ictiómetro: longitud total (LT: medida desde la punta del hocico, hasta el extremo final de la aleta caudal, con un error de medición de $\pm 0.1 \mathrm{~cm})$. A los tiburones machos se les determinó el estado reproductivo según la calcificación de los hemipenes, utilizando la clasificación para Mustelus canis de la Patagonía (Chiaramonte \& Pettovello 2000), la cual se describe a continuación:
- $\quad$ Estado I - Hemipenes cortos y suaves.

- Estado II - Hemipenes cortos y parcialmente calcificados.

- Estado III - Hemipenes totalmente calcificados, la base de los hemipenes puede ser rotada $180^{\circ}$ y vuelve a su posición original. Presencia de semen en los hemipenes.

Posterior a la toma de datos biométricos, a cada tiburón se colocó una marca de plástico enumerada, en su primera aleta dorsal. Luego del marcaje y antes de ser devuelto al mar, a cada tiburón se les aplicó una dosis de antiinflamatorio (Betametasona) para disminuir las probabilidades de infecciones. Por último, para los tiburones recapturados, se calculó el crecimiento, que corresponde a la diferencia entre la medida actual y la anterior, utilizando la fórmula: $\mathrm{C}=(\mathrm{LT} 2-\mathrm{LT} 1) /($ tiempo2-tiempo1 $)$.

Conteos subacuáticos visuales: Se llevaron a cabo seis conteos subacuáticos visuales de tiburones punta blanca de arrecife durante inmersiones SCUBA en Bahía Chatham. Para ello, se realizaron transectos paralelos a la línea de bajamar de la Bahía, de aproximadamente $500 \times 20 \mathrm{~m}$, utilizando la metodología propuesta por Ayling y Choat (2008). En cada conteo se registró la cantidad de tiburones marcados, así como la temperatura y profundidad relacionadas a cada individuo. Además, se registró: la duración de la inmersión, el tiempo efectivo destinado al conteo, temperatura y profundidad máxima durante la inmersión.

La abundancia relativa durante los conteos se estimó utilizando la siguiente fórmula:

$$
\text { Avistamiento por hora }=\frac{\mathrm{N}^{\mathrm{o}} \text { Individuos observados }}{\text { Tiempo efectivo }(\mathrm{min})} \times \text { hora }(60 \mathrm{~min})
$$

Análisis de datos: Se calculó la longitud total promedio y desviaciones estándar (DE) de los tiburones punta blanca, machos y hembras. Se utilizó un análisis de varianza no paramétrico de una vía (Kruskall-Wallis) para determinar diferencias entre la longitud total de machos y hembras utilizando el programa InfoStat (Di Rienzo et al. 2008). Con el mismo programa, se calculó el mínimo (min), máximo (max) y la desviación estándar (DE) de la longitud total. Se calculó el porcentaje de madurez de los machos según los estadios encontrados.

Finalmente, se estimó el tamaño poblacional para la especie en Bahía Chatham, utilizando el programa Capture (White et al. 1978) y seleccionando el modelo más apropiado $(\mathrm{Mt})$, 
este modelo corrige el sesgo temporal producto del muestro o condiciones climáticas que impiden muestreos entre unidades de tiempo iguales (Otis et al. 1978). Se realizaron dos estimaciones: la primera a partir de datos de las recapturas obtenidas exclusivamente durante las giras de marcaje nocturnas; y la segunda a partir de la fusión de las recapturas visuales, realizadas durante los conteos subacuáticos, con las recapturas obtenidas durante las giras nocturnas.

\section{RESULTADOS}

Marcaje de tiburones: Se realizaron nueve recorridos nocturnos, entre las 18:0022:00h, para el marcaje de tiburones punta blanca de arrecife. En los sitios de marcaje la profundidad y temperatura superficial del agua promedio fue de $10.9 \pm 1.9 \mathrm{~m}$ y $28.4 \pm 1.2^{\circ} \mathrm{C}$, respectivamente. Durante los nueve recorridos se marcaron 58 tiburones, de estos el $51.7 \%$ $(n=30)$ fueron hembras y el $48.3 \%$ machos $(n=28)$ para una proporción sexual de 1.07:1. La longitud total promedio de los tiburones marcados fue $101.8 \pm 12.1 \mathrm{~cm}$, con una longitud total máxima de $130.0 \mathrm{~cm}$ y una mínima de $71.0 \mathrm{~cm}$. La mediana de las longitudes totales de los tiburones machos $(103.5 \pm 21.33 \mathrm{~cm})$ y hembras $(100.1 \pm 11.2 \mathrm{~cm})$ no difirieron significativamente $(\mathrm{H}=1.78 ; \mathrm{p}=0.1818)$. Con respecto a la distribución de tiburones analizados, el $73 \%$ hembras y el $60 \%$ de los machos registraron longitudes totales 90 y $110 \mathrm{~cm}$. Mientras solo el $15 \%$ de las hembras y $25 \%$ de los machos presentaron longitudes entre $111 \mathrm{y}$ $130 \mathrm{~cm}$ (Fig. 1).

La mayoría de los machos marcados (57\%) se encontraban en el estado II o madurando (sub-adultos); mientras que, la menor proporción de los machos correspondió al estado I, inmaduros (jóvenes) (Fig. 2).

Durante las giras nocturnas de marcaje se recapturaron cinco individuos: cuatro marcados unos días antes de la recaptura y uno marcado más de tres meses antes de la recaptura (Cuadro 1). Para este último es estimó

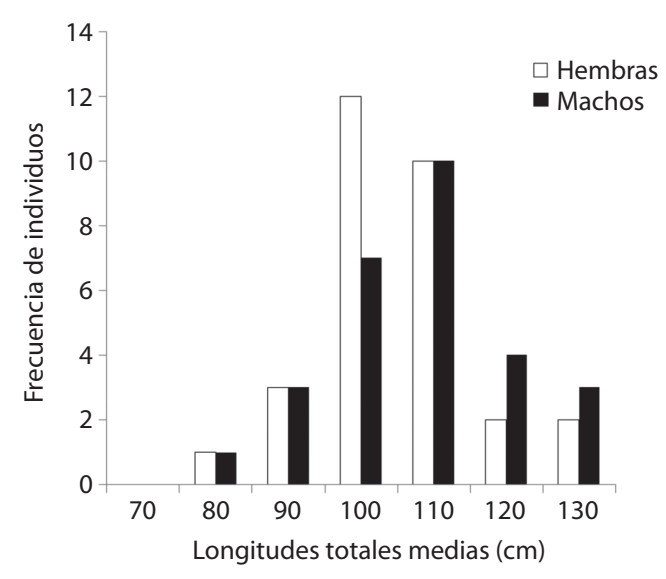

Fig. 1. Distribución de frecuencia por Longitud Total (LT) y por sexo de los tiburones punta blanca marcados $(n=58)$. Isla del Coco, Costa Rica, 2011.

Fig. 1. Frequency distribution of total length for whitetip reef shark by sex $(n=58)$. Cocos Island, Costa Rica, 2011.

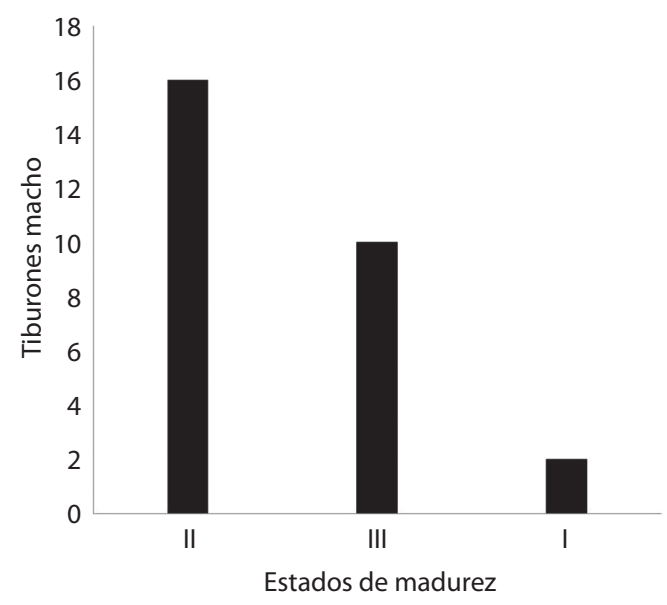

Fig. 2. Distribución de frecuencia por estados de madurez de los tiburones punta blanca machos marcados $(n=28)$. Isla del Coco, Costa Rica, 2011.

Fig. 2. Frequency distribution of sexual maturity stage for males of whitetip reef shark $(n=38)$. Cocos Island, Costa Rica, 2011.

el crecimiento mensual, el cual resultó ser de aproximadamente $1.9 \mathrm{~cm}$ por mes.

Conteos visuales subacuáticos: Se realizaron seis inmersiones diurnas, entre las 10:00-13:00, en Bahía Chatham. Durante los 
CUADRO 1

Recapturas de tiburones punta blanca de arrecife marcados en Bahía Chatham, Isla del Coco, Costa Rica

TABLE 1

Recaptures of tagged whitetip reef sharks in Chatham Bay, Coco's Island, Costa Rica

\begin{tabular}{cccc} 
Fecha de marcaje & Fecha de recaptura & Horas & Número de marca \\
$18-12-10$ & $26-12-10$ & $19: 15$ & 17 \\
$18-12-10$ & $26-12-11$ & $19: 30$ & 30 \\
$02-04-11$ & $04-04-11$ & $19: 30$ & 39 \\
$04-05-11$ & $06-04-11$ & $20: 31$ & 2 \\
\hline $26-12-10$ & $06-04-11$ & $21: 15$ & 30 \\
\hline
\end{tabular}

conteos, la temperatura y profundidad media fue de $28.5 \pm 1.3^{\circ} \mathrm{C}$ y $13.0 \pm 2.4 \mathrm{~m}$, respectivamente. La abundancia relativa de tiburones en Bahía Chatham fue de $49.5 \pm 10.4$ tiburones/h. Durante los conteos se hicieron 27 recapturas visuales, siendo posible identificar 19 tiburones de los 58 marcados previamente.

Con los datos de las recapturas obtenidas exclusivamente durante las giras nocturnas, la población se estimó en 408 , con intervalos de confianza (IC) del 95\% (IC =181-1054). Con la fusión de las recapturas visuales y las recapturas durante las giras nocturnas, fue de 94 ( $\mathrm{IC}=$ 76-128) con un 95\% de nivel de confianza.

\section{DISCUSIÓN}

Los tiburones punta blanca de arrecife se capturaron y marcaron en giras nocturnas, entre las 18:00-22:00h. Se realizaron intentos de capturas antes y después de este intervalo, sin embargo no resultaron ser efectivos. Es un tiburón nocturno, manifestando niveles más altos de actividad y tasa metabólica durante la noche respecto al día (Whitney et al. 2007, Fitzpatrick et al. 2011); sin embargo, resulta ser aún más activa durante el atardecer, siendo este comportamiento un resultado relacionado al forrajeo (Hobson 1963, Whitney et al. 2007). De la misma forma, la profundidad resultó ser un factor relevante para el marcaje ya que se logró capturar a los tiburones solo en zonas poco profundas $(10.9 \pm 1.9 \mathrm{~m})$ de Bahía Chatham.

El rango de la LT de los especímenes capturados durante las faenas nocturnas de marcaje coincide con lo reportado por Robbins (2006) para la Gran Barrera de Coral de Australia, en donde los autores reportaron tallas de capturas del tiburón punta blanca por parte de pescadores comerciales, entre los 70.0 y $140.0 \mathrm{~cm}$. Además, según el análisis de estados reproductivos en machos, se encontraron tiburones inmaduros (crías), madurando (jóvenes) y maduros (adultos), lo cual confirma que esta especie no posee una segregación ontogénica espacial bien definida, como sí se ha reportado para otras especies como $S$. lewini (Klimley 1987, Torres 2004, Zanella et al. 2009) y el tiburón toro, Carcharhinus leucas (Heupel \& Simpfendorfer 2008, McCord \& Lamberth 2009). A pesar de esto, la longitud total máxima registrada en el estudio está por debajo de los $213 \mathrm{~cm}$ reportada por Compagno et al. (1995) para esta especie. Esto puede ser consecuencia del arte de pesca utilizado para su captura (referente a tamaño de los anzuelos) o bien, a que los individuos más grandes y longevos se encuentren en aguas más profundas, ubicadas fuera de Bahía Chatham. Lanyon (2011) en las Islas Catalinas, Golfo de Papagayo, Costa Rica, reportó una relación directa entre la longitud total del tiburón punta blanca y la profundidad, sugiriendo que en aguas más profundas se encuentran los individuos más grandes. A su vez, Heupel et al. (2010) describieron como el tiburón gris de arrecife (Carcharhinus amblyrhynchos) en Ribbons Reef, Australia, mostró un cambio de patrones de movimiento, según la talla. Las crías y jóvenes tienen una mayor fidelidad a los sitios, mientras que los adultos 
tienden a desplazarse más. Por tanto, conforme van creciendo muestran una menor fidelidad a los sitios, comportamiento probablemente relacionado al forrajeo.

Durante el marcaje, también se obtuvo una proporción alta de recapturas, lo cual sugiere una alta fidelidad a los sitios de algunos individuos. Sin embargo, los datos no permitieron estimar debidamente el crecimiento mensual. Para poder describir de manera apropiada una curva de crecimiento para esta especie en el PNIC, se deberán llevar a cabo más estudios y marcar un mayor número de individuos. Esto debido a que el crecimiento en vertebrados marinos no es constante, sino que decrece con la edad (Di Benedetti \& Ramos 2004). El tiburón punta blanca de arrecife, comparado con otros tiburones, manifiesta una tasa de crecimiento inicial relativamente baja, aumentando un $38 \%$ de su talla de nacimiento durante el primer año (Robbins 2006). Otros tiburones, como el picudo (Rhizoprionodon taylori) tienen un crecimiento inicial mucho más rápido, aumentando hasta un $140 \%$ de su talla de nacimiento durante su primer año de vida (Simpfendorfer 1993, Robbins 2006).

Durante los conteos se reportó una abundancia relativa de tiburones punta blanca de arrecife en Bahía Chatham de 49.5 \pm 10.4 tiburones/h, lo cual es notablemente mayor a lo encontrado por Arauz et al. (2009) para Bahía Culebra y alrededores, cuyas estimaciones de abundancia relativa fueron entre $2.37 \pm 1.24$ tiburones/h en enero, y $12.77 \pm 11.16$ tiburones/ hora en junio. Distintos autores han indicado como la temperatura influye en la presencia y abundancia relativa del tiburón punta blanca. En el PNIC, durante los eventos de El Niño se encontró una disminución en los avistamientos del tiburón punta blanca (Sibaja 2008). En las Islas Catalinas, Golfo de Papagayo (Costa Rica) se observó que esta especie está presente cuando la temperatura estuvo entre los $25-30^{\circ} \mathrm{C}$ (Lanyon 2011). La temperatura promedio durante las inmersiones realizadas en Bahía Chatham fue de $28.5 \pm 1.3^{\circ} \mathrm{C}$; sin embargo, con el fin de monitorear esta población en el área se recomienda realizar conteos subacuáticos mensuales a largo del tiempo, y relacionar su abundancia con la temperatura del agua.

Los conteos subacuáticos confirmaron la alta fidelidad del tiburón punta blanca en Bahía Chatham, de hecho por lo menos el $32.8 \%$ de los tiburones marcados fueron re-observados durante las inmersiones. Esta especie utiliza distintos sitios de residencia con alta fidelidad (Randall 1977, Peñaherrera et al. 2011); Randall (1977) describe como estos sitios distan aproximadamente tres $\mathrm{km}$ entre ellos. Por lo tanto, es posible que los tiburones durante el día se dispersen entre los distintos sitios de residencia y se congreguen durante las primeras horas de la noche en aguas de Bahía Chatham para alimentarse. Esto explicaría también la alta variación obtenida entre las dos estimaciones del tamaño poblacional. Sin embargo, debido a la alta varianza reportada en la estimación durante los muestreos nocturnos (408.0, IC=181-1054), se recomienda aumentar el número de muestreos y marcar una mayor cantidad de individuos, con el fin de obtener datos más robustos.

\section{AGRADECIMIENTOS}

Al Parque Nacional Isla del Coco y al Área de Conservación Marina Isla del Coco por habernos apoyado durante las expediciones con recursos, personal y embarcaciones. A los funcionarios del Parque Nacional Isla del Coco que han participado activamente en la investigación, un agradecimiento especial a Maikel Pérez Montero y Keilor Morales por su ayuda durante la captura y manipulación de los tiburones; a Moisés Gómez por sus valiosos aportes en el método de captura. A Carlos Hiller por asistirnos durante la segunda expedición. Flying Sharks por brindarnos el apoyo financiero necesario para llevar a cabo esta investigación.

\section{RESUMEN}

Doce especies de tiburones habitan las aguas del Parque Nacional Isla del Coco (PNIC) y uno de los más abundantes es el tiburón punta blanca de arrecife, Triaenodon 
obesus. El presente estudio determinó la abundancia, tallas y estructura de la población de esta especie en la zona de Bahía Chatham, PNIC. Se utilizó el método de marcaje y recaptura y se realizaron conteos subacuáticos visuales, con el fin de determinar su abundancia relativa y contabilizar recapturas visuales. Los tiburones punta blanca de Bahía Chatham no presentan segregación ontogénica espacial definida; pero si una alta fidelidad a los a sitios de residencia dentro de esta bahía. La longitud total promedio de los tiburones marcados fue de $101.8 \pm 12.1 \mathrm{~cm}$, con una longitud total máxima de $130.0 \mathrm{~cm}$ y una mínima de $71.0 \mathrm{~cm}$. La mediana de las longitudes totales de los tiburones machos $(103.5 \pm 21.33 \mathrm{~cm})$ y hembras $(100.1 \pm 11.2 \mathrm{~cm})$ no difirieron significativamente $(H=1.78 ; \mathrm{p}=0.1818)$. La abundancia relativa en Bahía Chatham, PNIC fue de $49.5 \pm 10.4$ tiburones/h. El tamaño estimado de la población usando el método de de captura-recaptura fue de 408 (IC=181-1050).

Palabras claves: Parque Nacional Isla del Coco, Triaenodon obesus, Bahía Chatham, marcaje, conteos subacuáticos visuales, tiburones, Costa Rica

\section{REFERENCIAS}

ACMIC. 2002. La presentación de informes periódicos sobre la aplicación de la Convención del Patrimonio Mundial. Sección II: Estado de conservación de bienes específicos del Patrimonio Mundial. UNESCO, San José, Costa Rica.

Arauz, R., I. Zanella \& A. López. 2009. Caracterización de los elasmobranquios avistados en sitios de buceo, en el Golfo de Papagayo, Costa Rica. PRETOMA. San José, Costa Rica.

Ayling, A. \& J. Choat. 2008. Abundance patterns of reef sharks and predatory fishes on differently zoned reefs in the offshore Townsville region: Final Report to the Great Barrier Reef Marine Park Authority, Queensland, Australia.

Chiaramonte, G. \& A. Pettovello. 2000. The biology of Mustelus schmitti in southern Patagonia, Argentina. J. Fish Biol. 57: 930-942.

Compagno, L., F. Krupp \& W. Schneider. 1995. Tiburones, p. 647-743 In: W. Fischer, F. Krupp, W. Schneider, C. Sommer, K. Carpenter \& V. Niem (eds). Pacífico centro-oriental. Volumen II. Vertebrados- Parte I. Guía FAO para la identificación de especies para los fines de la pesca. FAO, Roma, Italia.

Cortés, J. 2008. Historia de la investigación marina de la Isla del Coco, Costa Rica. Rev. Biol. Trop. 56 (Supl. 2): 1-18.

Di Benedetti, A.P. \& R.M. Ramos. 2004. Biology of the marine tucuxi dolphin (Sotalia fluviatilis) in south-eastern Brazil. J. Mar. Biol. Assoc. UK 84: $1245-1250$.
Di Rienzo, J.A, F. Casanoves, M. Balzarini, L. Gonzalez, M. Cuadroda \& C. Robledo. 2008. InfoStat versión 2008. Grupo InfoStat, FCA, Universidad Nacional de Córdoba, Argentina.

Fitzpatrick, R., K. Abrantes, J. Seymour \& A. Barnett. 2011. Variation in depth of whitetip reef sharks: does provisioning ecotourism change their behaviour? Coral Reefs 30: 569-577.

Garrison, G. 2005. Peces de la Isla del Coco. 2da Ed. Edit. INBio, Santo Domingo de Heredia, Costa Rica.

Heupel, M. \& C. Simpfendorfer. 2008. Movements and distribution of young bull sharks (Carcharhinus leucas) in a variable estuarine environment. Aquat. Biol. 1: 277-289.

Heupel, M., C. Simpfendorfer \& R. Fitzpatrick. 2010. Large-scale movement and reef fidelity of grey reef sharks. PLoS ONE 5(3): e9650.

Hobson, E. S. 1963. Feeding behavior in three species of sharks. Pac. Sci. 17: 171-194.

Klimley, A. P. 1987. The determinants of sexual segregation in the scalloped hammerhead shark, Sphyrna lewini. Environ. Biol. Fish. 18:27-40.

Lanyon, A. 2011. Influencia de la temperatura y profundidad del mar en la distribución y abundancia del tiburón puntablanca Triaenodon obesus (Carcharhiniformes: Carcharhinidae) en el Golfo de Papagayo, Costa Rica. Informe final BSc, Univ. Latina, San José, Costa Rica.

McCord, M \& S. Lamberth. 2009. Catching and tracking the world's largest Zambezi (bull) shark Carcharhinus leucas in the Breede Estuary, South Africa: the first 43 hours. Afr. J. Ecol. 31: 107-111.

Onca Natural \& ACMIC. 2007. Plan de Manejo del Parque Nacional Isla del Coco. Ministerio del Ambiente y Energía, Sistema Nacional de Áreas de conservación, Área de Conservación Marina Isla del Coco, San José, Costa Rica.

Otis, D.; K.P. Burnham; G.C. White \& D.R. Anderson. 1978. Statistical Inference from Capture Data on Closed Animal Populations. Wildlife Monogr. 62: 1-135.

Peñaherrera, C., A. Hearn \& A. Kuhn. 2011. Diel use of a saltwater creek by white tip reef sharks Triaenodon obesus (Carcharhinidae) in Academy Bay, Galapagos Islands. Islas Galápagos, Ecuador. Sin publicar.

Randall, J. 1977. Contribution to the biology of the whitetip reef shark (Triaenodon obesus). Pac. Sci. 31: 143-164.

Robbins, W. 2006. Abundance, demography and population structure of the grey reef shark (Carcharhinus amblyrhynchos) and the whitetip reef shark (Triaenodon obesus) (Fam. Carcharhinidae). Tesis $\mathrm{PhD}$, James Cook Univ., Townsville, Australia. 
Sibaja, J. 2008. Tendencias espacio-temporales de los avistamientos de fauna marina en los buceos turísticos (Isla del Coco, Costa Rica). Rev. Biol. Trop. 56 (Supl. 2): $113-132$.

Simpfendorfer, C. 1993. Age and growth of the Australian sharpnose shark, Rhizoprionodon taylori, from north Queensland, Australia. Environ. Biol.Fish 36: 233-24.

Sinergia 69. 2002. Anexo V: Caracterización de las bahías de Chatham y Wafer e inventario preliminar de especies marinas. Área de Conservación Marina Isla del Coco (ACMIC). Proyecto: Estudio de factibilidad y diseño para la construcción de infraestructura y servicios de apoyo en el Parque Nacional Isla del Coco. Sinergia, San José, Costa Rica.

Torres, A. 2004. Distribución, abundancia y hábitos alimentarios de juveniles del tiburón martillo Sphyrna lewini, Griffith y Smith (SPHYRNIDAE) en la costa de Sinaloa, México durante el evento El Niño 1997. 98. Tesis MSc, Univ. Mar. Oaxaca, México.
White, G., K. Burnham, D. Otis \& D. Anderson. 1978. Program Capture. Utah State Univ. Press. Logan, Utah, EUA.

Whitney, N. \& P. Motta. 2007. Cleaner host posing behavior of whitetip reef sharks (Triaenodon obesus) in a swarm of hyperiid amphipods. Coral Reefs: 363 .

Whitney, N., H. Pratt, \& J. Carrier. 2004. Group courtship, mating behaviour and siphon sacfunction in the whitetip reef shark, Triaenodon obesus. Anim. Behav. 68: 1435-1442.

Whitney, N., Y. Papastamatiou, K. Holland \& C. Lowe. 2007. Use of an acceleration data logger to measure diel activity patterns in captive whitetip reef sharks, Triaenodon obesus. Aquat. Liv. Resour. 20: 299-305.

Zanella, I., A. López \& R. Arauz. 2009. Caracterización de las descargas del tiburón martillo, Sphyrna lewini, en un área de crianza ubicada en el Golfo de Nicoya, Costa Rica. Rev. Cienc. Mar. Cost. 1:175-195. 